\title{
Energy-Efficient RIS-Assisted Satellites for IoT Networks
}

\author{
Kürşat Tekbıyık, Graduate Student Member, IEEE, Güneş Karabulut Kurt, Senior Member, IEEE, \\ Halim Yanikomeroglu, Fellow, IEEE
}

\begin{abstract}
The use of satellites to provide ubiquitous coverage and connectivity for densely deployed Internet of Things (IoT) networks is expected to be a reality in emerging 6G networks. Yet the low battery capacity of IoT nodes constitutes a problem for their direct connectivity to satellites, which are located at altitudes of up to $2000 \mathrm{~km}$. In this paper, we propose a novel architecture involving the use of reconfigurable intelligent surface (RIS) units to mitigate the path loss associated with long transmission distances. These RIS units can be placed on satellite reflectarrays, and, when used in broadcasting and beamforming, they can provide significant gains in signal transmission. This study shows that RIS-assisted satellites can provide up to $10^{5}$ times higher downlink and achievable uplink rates for IoT networks.
\end{abstract}

Index Terms-Reconfigurable intelligent surfaces, LEO satellites, energy-efficient IoT networks.

\section{INTRODUCTION}

Vendors and business advisory companies expect that around 100 billion devices will be connected in a massive ecosystem by 2025 [1, 2]. Internet of Things (IoT) networks are expected to grow by approximately 20 percent compound annual growth rates [3]. By leveraging ubiquitous IoT networks, it is possible to increase the efficiency in industries such as transportation, health, and maritime. Another prominent feature of massive IoT networks is that they improve quality of life. Although ubiquitous and massive IoT networks have a broad range of advantages for industry and human life, backhaul of these ultra-massive networks is still challenging issue. Furthermore, Internet access is still limited in some parts of the world. For instance, 4 billion people do not have an Internet connection [4]. To expand coverage area, supporting IoT services with satellite networks is a prominent research topic for both academia and industry.

\section{A. Related Works}

Several recent works have proposed for the use of geostationary orbit (GEO) satellites in narrowband-IoT applications [5, 6]. However, GEO satellites suffer high path losses and long delays due to their considerable distance from the Earth. To eliminate the drawback of GEO satellites, low-Earth orbit (LEO) satellite constellations, which orbit the Earth closer than GEO satellites, were introduced for IoT networks

K. Tekbiyık and G.K. Kurt are with the Department of Electronics and Communications Engineering, İstanbul Technical University, İstanbul, Turkey, e-mails: \{tekbiyik, gkurt\}@itu.edu.tr

G. Karabulut Kurt is also with the Department of Electrical Engineering, Polytechnique Montréal, Montréal, Canada, e-mail: gunes.kurt@polymtl.ca

H. Yanikomeroglu is with the Department of Systems and Computer Engineering, Carleton University, Ottawa, Canada, e-mail: halim@sce.carleton.ca in $[7,8]$. LEO satellites require less transmission power to maintain a requisite signal-to-noise ratio (SNR) for proper communication, but they have drawbacks as well. In particular, the high velocities of LEO satellites make the steering antennas and satellite tracking systems essential. Because IoT devices have low battery capacity and low computing power, they cannot track LEO satellites and run high-complexity signal processing algorithms in the receiver unit. Thus, IoT devices need low complexity transceivers and energy-efficient signal processing and tracking methods. In order to track satellites and decrease computational cost at receiver in an energyefficient way, we propose using reconfigurable intelligent surfaces (RISs) in LEO satellites in this study. RISs intelligently adjust the phase shifts of elements in order to maximize the received power [9]. Furthermore, RISs are also advantageous because they consume less power and have lower hardware costs than conventional arrays. This is because conventional arrays have bulky feeding networks, which result in significant power losses. By contrast, RIS phase control mechanisms use simple PIN diodes [10]. But, the most appealing feature of RISs is that they are entirely comprised of passive circuit elements and require no complex processing or coding. The effectiveness of RISs has been demonstrated by experimental measurements in [10, 11]. Metasurface technologies, which include RISs, have also recently been proposed for deep space communications in [12-15]. By utilizing RISs, it is possible to improve performance in terms of power consumption and/or diversity gain.

The installation of RIS units on LEO satellites does not require serious hardware improvements, as RISs have a simple hardware structure consisting of passive circuit elements [16]. Moreover, the hardware complexity of IoT devices can be reduced as RISs allow signal processing to be performed in the transmission environment rather than on the devices [9]. Thus, battery-constrained IoT devices can operate for a longer duration. Furthermore, RISs can enable energy-efficient wireless communication while maintaining the same quality of service (QoS) level [17]. Our previous work [16] has also demonstrated that error probability can be reduced in LEO inter-satellite links by utilizing RISs.

\section{B. Motivations}

As the number of IoT applications and devices increases exponentially, the need for ubiquitous Internet coverage is becoming increasingly apparent. LEO satellites offer a promising solution for this. With a high number of satellites in nearEarth constellations, it will become possible to ubiquitously 


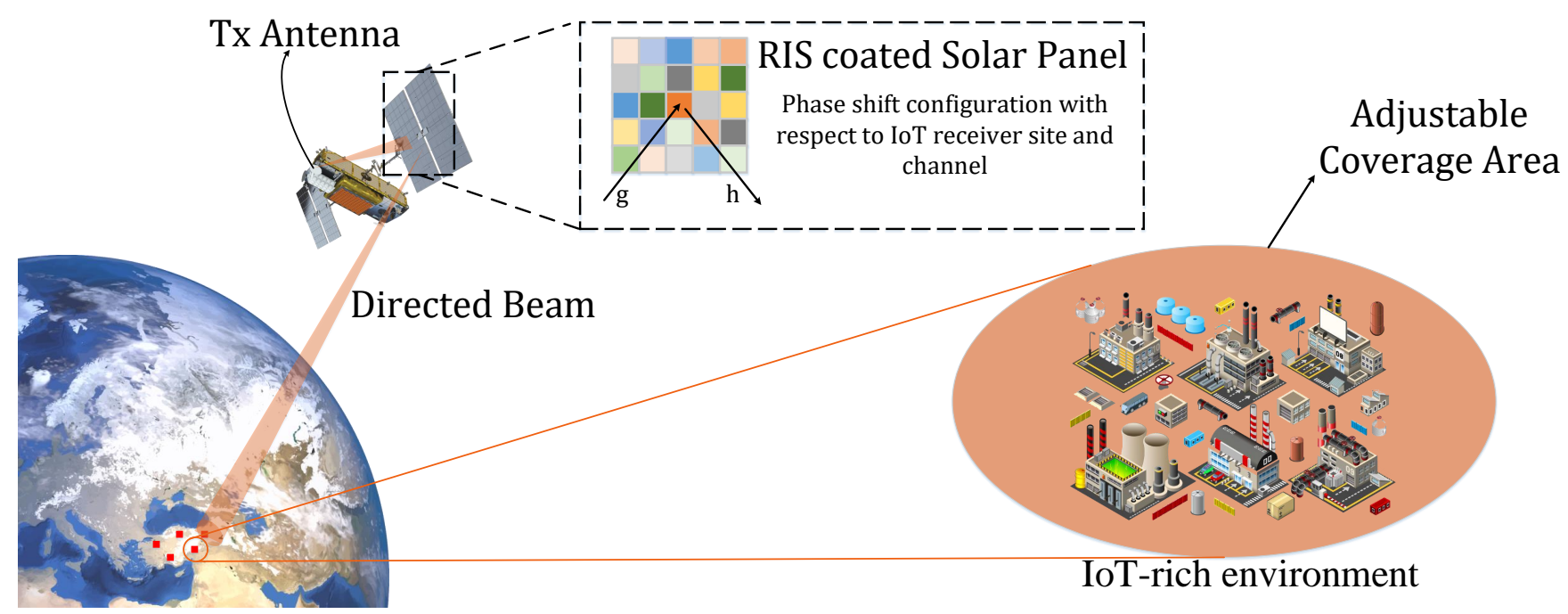

Fig. 1. It is possible to enhance the QoS for satellite-IoT systems by utilizing RISs. Therefore, the required power can be reduced for the same data rate and error probability.

and continuously service users. The provision of IoT services via LEO networks has been under investigation not only by the research community but also by standards organizations, such as 3GPP, and private companies, such as Satelliot. Especially with the $5 \mathrm{G}$ standards, following the 3GPP TR 36.763 [18], IoT deployments involving satellites will be a reality in the near future, not only for rural areas that do not get sufficient coverage but also for improving the capacity in highly populated dense deployments in mega-cities.

As IoT devices have limited battery life, any boost in the link budget will be useful in satellite networks. To address this issue in this study, we propose the use of RISs on the receiver end to improve received power levels. RIS units, which are also called meta-atom, are composed of metasurfaces that are controlled in real time to adjust the reflection phases [9]. The potential of RISs has already been discussed in recent works, including $[17,19]$. In this study, we propose the use of RIS on satellites that can be positioned jointly with a reflective array as illustrated in Fig. 1. Due to the large surface area under satellite solar panels, numerous RIS units can be installed. By configuring the phase shift for each meta-atom, the incident wave can be scattered or beamformed to target users. Furthermore, by properly selecting phase shifts, the coverage area can be adaptively adjusted with respect to the stochastic geometry of IoT networks. In investigating the use of RISs in satellite IoT systems, we show that it is possible to reduce power consumption and hardware costs of IoT devices. This is made possible by the fact that RISs can increase the received SNR and reduce computational complexity by eliminating the need for complex signal processing applications at the receiver end.

\section{Contributions}

The contributions of the paper on the way to provide ubiquitous and dense connectivity demanded by $6 \mathrm{G}$ and beyond are summarized here:

C1 A novel architecture is proposed for IoT networks based on the use of RIS units with LEO satellites. These are RIS units can be placed under the solar panels or replaced with reflect arrays.

C2 The SNR levels of LEO satellites for supporting IoT networks is quantified, taking into consideration broadcasting and beamforming modes based on transmission characteristics, including carrier frequency.

C3 Through extensive numerical results, we quantify the potential reduction of the transmit power using a realistic transmission model. Based on our analysis, we suggest design guidelines for future IoT networks served by LEO satellites.

\section{Outline}

The rest of this paper is organized as follows. Section II introduces the free-space path loss models for RIS-assisted broadcasting and RIS-assisted beamforming schemes as well as a case without RIS. In Section III, the system model is described for RIS-assisted LEO satellites for IoT networks. Section IV presents extensive numerical results and observations for non-RIS satellites and RIS-assisted satellites with both broadcasting and beamforming schemes. Open issues are discussed in Section V. Finally, the study is concluded in Section VI.

\section{Preliminaries}

Here we discuss the mathematical and physical preliminaries for RIS-assisted satellites for IoT networks. We begin with an overview of free-space path loss for conventional satellite systems. Then, we introduce a path loss model for RIS-assisted wireless communications. In what follows, the notation is given for the downlink; however, the notation can be generalized for the uplink case in a straightforward manner.

\section{A. Free-Space Path Loss}

The ratio of the received and transmitted powers in a link between two isotropic antennas can be given by free-space 


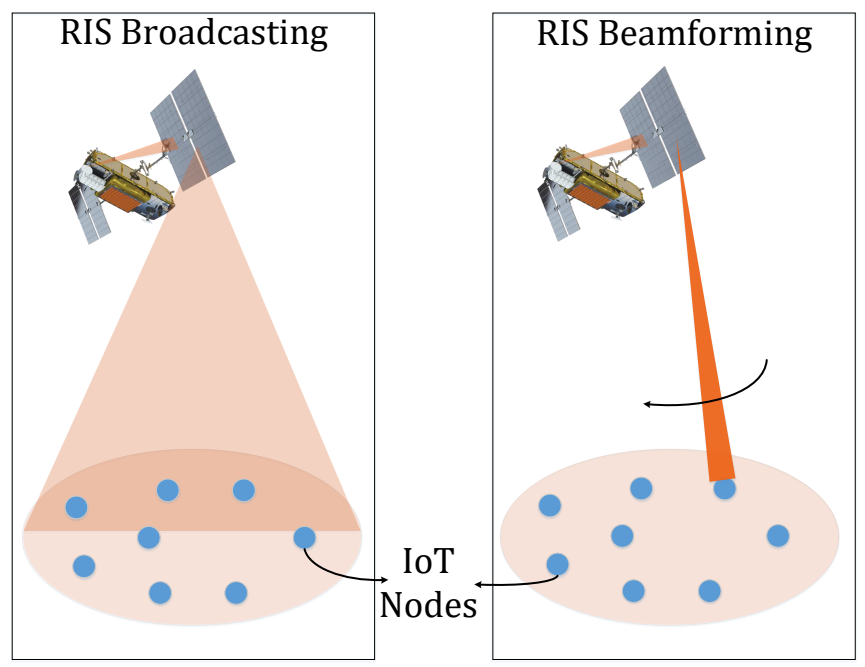

(a)

Fig. 2. RIS-assisted satellites can serve in two modes: (a) RIS broadcasting and (b) RIS beamforming. In the former, the RISs scatter the incident wave; in the latter, the RISs perform specular reflection.

path loss, which is defined as follows:

$$
L_{\mathrm{FS}}=(4 \pi d / \lambda)^{\alpha},
$$

where $\alpha$ and $\lambda$ denote the path loss exponent and wavelength, respectively. $d$ is the distance between the satellite and ground station, which can be obtained accordingly:

$$
d=-r_{e} \sin (\varphi)+\sqrt{r_{e}^{2} \sin ^{2}(\varphi)+h_{\mathrm{sat}}^{2}+2 r_{e} h_{\mathrm{sat}}},
$$

where $r_{e}, h_{\mathrm{sat}}$, and $\varphi$ represent the radius of the Earth, the altitude of the satellite, and the elevation angle between the ground station and satellite, respectively. The path loss is proportional to $d^{\alpha}$; therefore, the elevation angle plays an important role in the path loss.

\section{B. Path Loss Models for RIS-Assisted Communications}

Here we describe the path loss models for RIS-assisted wireless communications in broadcasting and beamforming cases.

We begin with the broadcasting case as illustrated in Fig. 2(a). If the transmitter is in the near-field of RIS, and the surface is at least ten times larger electrically than the wavelength, $\lambda$, the surface scatters the incident spherical wave. Scattering the incident wave creates a large beam, which can cover multiple ground stations at the same time. As the transmitter antenna is relatively near to the RIS deployed on the satellite, the scattering paradigm should be considered in the case of electrically large surfaces. The path loss model for RISassisted wireless communications in the case of broadcasting is the following [11]:

$$
P L_{\mathrm{BC}} \approx \frac{(4 \pi)^{\alpha}\left(d_{t x}+d_{r x}\right)^{\alpha}}{G_{t} G_{r} \lambda^{\alpha} A^{\alpha}},
$$

where $d_{t x}$ and $d_{r x}$ are the distances from the transmitter antenna to the RIS and from the RIS to the receiver antenna,

respectively. As $d_{r x} \gg d_{t x}$, the distance between the transmitter antenna and the RIS can be omitted and $d_{r x}=d$. Then, the expression can be written as follows:

$$
P L_{\mathrm{BC}} \approx \frac{(4 \pi d)^{\alpha}}{G_{t} G_{r} \lambda^{\alpha} A^{\alpha}} .
$$

Here, $G_{t}$ and $G_{r}$ denote the antenna gain for the transmitter and receiver antennas, respectively. $A$ is the amplitude of the reflection coefficient of meta-atoms. It should be noted that the free-space path loss for the RIS broadcasting scheme is not dependent on the number of RIS elements, as seen in (4). In this scheme, the RIS scatters the incident wave. Thus, while a wider coverage is obtained, the amount of energy per area is slightly lower.

But, the transmitted wave can also be focused on the receiver in order to improve the received signal quality, as depicted in Fig. 2(b). Both the transmitter and receiver can be in the far-field of the RIS, or one of them can be in the nearfield of the RIS. The latter is the more appropriate model for satellite IoT systems, which includes a transmitter near to the RIS. Therefore, we utilize the near-field beamforming scheme for the RIS. In other ways, it can be said that RIS operates as a specular reflection [20]. The path loss model for near-field beamforming scheme in an RIS-assisted satellite is given as follows [11]:

$$
P L_{\mathrm{BF}}=\frac{64 \pi^{3}}{G_{t} G_{r} d_{x} d_{y} \lambda^{\alpha} A^{\alpha}\left|\sum_{n=1}^{N} \frac{\sqrt{F_{n}^{\text {combine }}}}{d_{t x} d_{r x}}\right|^{\alpha}},
$$

where $N$ is the number of meta-atoms. $d_{x}$ and $d_{y}$ are the size of each unit cell along the $\mathrm{x}$-axis and $\mathrm{y}$-axis, respectively. $F_{n}^{\text {combine }}$ denotes the accounted normalized power radiation pattern on the received signal power, which can be described accordingly:

$F_{n}^{\text {combine }}=F^{t x}\left(\theta_{n}^{t x}, \beta_{n}^{t x}\right) F\left(\theta_{n}^{t}, \beta_{n}^{t}\right) F\left(\theta_{n}^{r}, \beta_{n}^{r}\right) F^{r x}\left(\theta_{n}^{r x}, \beta_{n}^{r x}\right)$,

where $F\left(\theta_{n}, \beta_{n}\right)$ shows the normalized radiation pattern for the elevation angle $\theta_{n}$ and the azimuth angle $\beta_{n}$ between the RIS and transmitter antenna (or receiver antenna). The normalized radiation pattern for the elevation angle $\theta$ and azimuth angle $\beta$ is defined as follows [21]:

$$
F(\theta, \beta)=\left\{\begin{array}{r}
\cos ^{3}(\theta), \theta \in\left[0, \frac{\pi}{2}\right], \beta \in[0,2 \pi] \\
0, \theta \in\left(\frac{\pi}{2}, \pi\right], \beta \in[0,2 \pi]
\end{array}\right.
$$

It is worth noting that in order to maximize the received power, the transmitter antenna has to be deployed to the satellite such that the transmitter antenna's normal line is orthogonal to the surface. Namely, $\theta_{n}^{t x}=\theta_{n}^{t}=0$ for all unit cells. Without loss of generality, it is assumed that $\theta_{n}^{r x}=\theta_{n}^{r}=\frac{\pi}{2}-\varphi$ and $d_{r x} \gg d_{t x}$. As expected, selecting $\theta_{n}^{t x}$ and $\theta_{n}^{t}$ is a design issue and it can be adjusted accordingly. The most important issue in the RIS beamforming scheme is that the loss gradually decreases with the increasing number of RIS elements.

\section{Rain Attenuation}

Rain attenuation is a major propagation impairment for satellite systems. Rain can cause scattering and absorption of 
waves propagating through the atmosphere. Rain attenuation is described by ITU-R P.618-13 as [22]:

$$
P L_{\text {rain }}=\xi_{R} L_{E}(\mathrm{~dB}),
$$

where $\xi_{R}$ and $L_{E}$ refer respectively to the specific frequencydependent coefficient, described by ITU-R P.838 [23], and the effective path length. First, we introduce the steps to find the value of $\xi_{R}$.

$$
\xi_{R}=k\left(R_{0.01}\right)^{\nu} \quad(\mathrm{dB} / \mathrm{km}),
$$

where $R_{0.01}$ is the rainfall rate, which can be obtained from ITU-R P.837 [24] for the location of a ground station. $k$ and $\nu$ denote the frequency-dependent coefficients given in ITU-R P.838 [23] as follows:

$$
\begin{aligned}
& k=\left[k_{H}+k_{V}+\left(k_{H}-k_{V}\right) \cos ^{2}(\varphi) \cos (2 \tau)\right] / 2 \\
& \nu=\left[k_{H} \nu_{H}+k_{V} \nu_{V}+\left(k_{H} \nu_{H}-k_{V} \nu_{V}\right) \cos ^{2}(\varphi) \cos (2 \tau)\right] / 2 \mathrm{k},
\end{aligned}
$$

where $\tau=\frac{\pi}{4}$ for circular polarization, and all coefficients are listed in Table I for $4.25 \mathrm{GHz}$ and $10.5 \mathrm{GHz}$.

In order to find the effective path length $L_{E}$, which is

$$
L_{\mathrm{E}}=L_{\mathrm{R}} v_{0.01} \quad(\mathrm{~km}),
$$

where $v_{0.01}$ is the vertical adjustment factor modeled as follows:

$v_{0.01}=\frac{1}{1+\sqrt{\sin (\varphi)}\left(31\left(1-\mathrm{e}^{-(\varphi /(1+\chi))}\right) \frac{\sqrt{L_{R} \xi_{R}}}{f^{2}}-0.45\right)}$.

$f$ is the frequency in $\mathrm{GHz}$ and $\chi$ is defined as [25]:

$$
\chi= \begin{cases}36-\mid \text { latitude } \mid, & \mid \text { latitude } \mid<36^{\circ} \\ 0, & \text { otherwise. }\end{cases}
$$

Also, $L_{R}$ is described as:

$$
L_{R}= \begin{cases}\frac{L_{G} r_{0.01}}{\cos (\varphi)}, & \tan ^{-1}\left(\frac{h_{R}-h_{S}}{L_{G} r_{0.01}}\right)>\varphi \\ \frac{\left(h_{R}-h_{S}\right)}{\sin (\varphi)}, & \text { otherwise. }\end{cases}
$$

where $h_{S}$ is the altitude of the ground station in $\mathrm{km}$. Also, $L_{G}$ is defined as:

$$
L_{G}= \begin{cases}L_{S} \cos (\varphi), & h_{R}-h_{S}>0 \\ 0, & h_{R}-h_{S} \leq 0\end{cases}
$$

where $L_{S}$ is a slant-path length calculated as follows:

$$
L_{S}= \begin{cases}\frac{2\left(h_{R}-h_{S}\right)}{\sqrt{\sin ^{2}(\varphi)+\frac{2\left(h_{R}-h_{S}\right)}{r_{e}}}+\sin (\varphi)}, & \varphi \leq 5^{\circ} \\ \frac{h_{R}-h_{S}}{\sin (\varphi)}, & \varphi>5^{\circ}\end{cases}
$$

where $h_{R}$ is the effective height of the rain described by ITUR P.839 [26] as:

$$
h_{R}=h_{0}+0.36 \quad(\mathrm{~km}),
$$

where $h_{0}$ is the mean $0^{\circ}$ isotherm height above mean sea level, which is a site-specific value.

\section{LEO SATELLITE-ENABLED IOT NETWORKS}

LEO satellite constellations have emerged as a popular way of assisting global IoT networks with reasonable delays [27]. Because of the expense of the gateway system and inflexible infrastructure, direct access is desired in LEO satellite IoT systems [28]. In this section, we describe a novel energyefficient system model for satellite communications with a transmitter antenna ${ }^{1}$ in the near-field of the RIS on a satellite. In order to maximize the received power, the transmitter antenna needs to be aligned with the normal line of the RIS, which makes the angle between the normal line and antenna beam zero. Thus, the normalized radiation pattern takes the maximum value as observed in (7). Furthermore, the distance $d_{t x}$ between the transmit antenna and the RIS is very short compared to the distance to ground station. Hence, the wireless channel between the transmitter and RIS can be omitted. The received signal $y$ can be introduced as follows:

$$
y=\sqrt{\frac{P_{t}}{P L}} \mathbf{g}^{\mathrm{T}} \mathbf{\Phi} \mathbf{h} x+w,
$$

where $x$ and $w$ stand for the transmitted signal with power $P_{t}$ and additive white Gaussian noise (AWGN) at the receiver, respectively. The noise term, $w$, can be assumed to be distributed with $\mathcal{C N}\left(0, N_{0}\right)$. $\mathbf{h}$ is the channel coefficient vector for the link between the RIS and receiver, such that $\mathbf{h}=\left[h_{1}, h_{2}, \ldots, h_{N}\right] . \mathbf{g}$ stands for the channel coefficient for the link between the transmitter and RIS. As the transmit antenna is close to the RIS, we can ignore the channel effects in between the transmitter and RIS. Therefore, $\mathrm{g}$ can be selected as the vector of all ones such that $\mathrm{g}=\mathbf{1}_{N}$. It should be noted that satellite communications generally have line-of-sight (LOS) propagations, and thus, a Rician fading model is employed in satellite channel models [29]. We therefore assume that the channel coefficients follow a Rician distribution. As in [30], the Rician factor of the channel is selected as $K=10$. $\boldsymbol{\Phi}$ denotes the responses of the RIS element can be expressed as follows:

$$
\Phi=\operatorname{diag}\left\{A_{1} \mathrm{e}^{j \phi_{1}}, \ldots, A_{N} \mathrm{e}^{j \phi_{N}}\right\},
$$

where $A_{i}$ and $\phi_{i}$ are the amplitude and phase response of $i$ th RIS element, respectively. Throughout this study, RISs are assumed to be lossless. Therefore, $A_{i}=A=1, \forall i$.

Next, regarding the received signal, the instantaneous SNR $\gamma$ can be given as follows:

$$
\gamma=\frac{\left|\mathbf{g}^{\mathrm{T}} \Phi \mathbf{h}\right|^{2} P_{t}}{N_{0} P L},
$$

where $P L$ represents the total loss, including free-space path $\operatorname{loss}^{2}$ and rain attenuation. As we can see in (20), the increased number of RIS elements improves the received SNR value. However, it should be emphasized that the increase in SNR values with respect to the number of RIS elements heavily depends on RIS design, as detailed above. Therefore, this work

\footnotetext{
${ }^{1}$ It should be noted that it is a receiver antenna for uplink communications.

${ }^{2}$ It can be either RIS-assisted wireless communications or not. For RISassisted communications, $P L_{\mathrm{BC}}$ and $P L_{\mathrm{BF}}$ are employed for RIS broadcasting and RIS beamforming, respectively. By contrast, it is equal to $P L_{\mathrm{FS}}$ in case of wireless communications without RIS.
} 
TABLE I

FREQUENCY-DEPENDENT COEFFICIENTS WHICH USED IN RAIN ATTENUATION CALCULATIONS FOR CIRCULAR POLARIZATION (ITU-R REC. P.838).

\begin{tabular}{lcc}
\hline \hline Coefficients & $\mathbf{4 . 2 5} \mathbf{G H z}$ & $\mathbf{1 0 . 5} \mathbf{G H z}$ \\
\hline$k_{H}$ & $7.3420 \mathrm{e}-4$ & $1.1926 \mathrm{e}-2$ \\
$k_{V}$ & $6.8259 \mathrm{e}-4$ & $1.0526 \mathrm{e}-2$ \\
$\nu_{H}$ & 1.1489 & 1.2602 \\
$\nu_{V}$ & 1.1034 & 1.2469 \\
Polarization & Circular & Circular \\
\hline$k$ & $7.084 \mathrm{e}-4$ & $1.123 \mathrm{e}-2$ \\
$\nu$ & 1.127 & 1.254 \\
\hline \hline
\end{tabular}

TABLE II

LOCATION-SPECIFIC COEFFICIENTS WHICH USED IN RAIN ATTENUATION CALCULATIONS FOR CIRCULAR POLARIZATION (ITU-R REC. P.838 \& ITU-R REC. P.839).

\begin{tabular}{lc}
\hline \hline Parameters & Values \\
\hline Location & Istanbul, Turkey \\
Latitude & $41^{\circ} \mathrm{N}$ \\
Longitude & $29^{\circ} \mathrm{E}$ \\
$h_{0}$ & $2.53 \mathrm{~km}$ \\
$R_{0.01}$ & 31.119 \\
$h_{S}$ & $1 \mathrm{~m}$ \\
$h_{\text {sat }}$ & $800 \mathrm{~km}$ \\
$r_{e}$ & $6371 \mathrm{~km}$ \\
\hline \hline
\end{tabular}

employs an RIS prototype, and its model is given in [10], rather than using a hypothetical RIS model.

For the free-space path loss calculation, (1) is utilized for satellite communications in non-RIS case. In RIS-assisted case, there two different modes, which are related to the dimension of the RIS element unit. For the RIS-assisted broadcasting scheme, (4) gives the free-space path loss, while (5) is the free-space path loss expression for RIS-assisted beamforming. Considering the SNR, the achievable data rate can be expressed as follows:

$$
R=\log _{2}(1+\gamma) \quad(\text { bits/s/Hz). }
$$

Due to the rise in the SNR value with an increasing number of RIS elements, the achievable rate also improves.

\section{NumericAl RESUlts AND Discussion}

In this section, we present a comprehensive simulation and discuss simulation results. First, we focus on the downlink capacity for satellite IoT systems. Then, we investigate the uplink capacity of satellite-supported IoT communications.

\section{A. Simulation Parameters}

We consider the simulation results for the two prominent bands for satellite IoT systems, namely C- and X-band. In simulations, the path loss model given in (1) was used for the case without RISs, while the models given in (4) and (5) are for RIS broadcasting and RIS beamforming, respectively.

As the rain attenuation changes significantly with respect to the operating frequency, it is important to properly acquire the frequency-dependent coefficients such as $k$ and $\nu$. For example, $k$ is found for $\mathrm{C}$ - and $\mathrm{X}$-band as $7.084 \mathrm{e}-4$ and $1.123 \mathrm{e}-2$, respectively. Besides, $\nu$ is equal to 1.127 and 1.254 for $\mathrm{C}$ - and $\mathrm{X}$-band, respectively. It is important to note that these values are valid for circular polarization, i.e. $\tau=\frac{\pi}{4}$. All frequency-dependent coefficients are given in Table I.

Also, in the simulations, we assume that the ground stations (i.e. IoT devices) are located in Istanbul, Turkey, which is located at $41^{\circ}$ north latitude and $29^{\circ}$ east longitude. Sitespecific coefficients such as the mean $0^{\circ}$ isotherm height above mean sea level $h_{0}$ and the rainfall rate $R_{0.01}$ is found as 2.53 $\mathrm{km}$ and 31.119 for Istanbul, Turkey. These parameters are summarized in Table II. As we investigate the LEO satellites, the satellite altitude $h_{\text {sat }}$ is chosen as $800 \mathrm{~km}$.

Other crucial factors to consider are the characteristics of the transmitter and receiver antennas and the RIS units. In the simulations, we employed the antennas and RISs given in [11]. These antennas have the normalized radiation pattern $F(\theta, \beta)$ that can be defined as follows ${ }^{3}$ :

$$
F^{t x}(\theta, \beta)= \begin{cases}\cos ^{13}(\theta), & f=4.25 \mathrm{GHz} \text { (C-band) } \\ \cos ^{62}(\theta), & f=10.5 \mathrm{GHz} \text { (X-band). }\end{cases}
$$

Furthermore, antenna gains are given as $14.5 \mathrm{~dB}$ and $21 \mathrm{~dB}$ for C-band and X-band antennas, respectively. As discussed above, the distance between the transmit antenna and the surface is short. Hence, $d_{t x}$ is selected as $1 \mathrm{~m}$ to keep the near-field condition for both bands. $d_{r x}$ is equal to the distance given by (2). In addition, RIS elements are square and their edge lengths are $0.012 \mathrm{~m}$ and $0.01 \mathrm{~m}$ for $4.25 \mathrm{GHz}$ and 10.5 $\mathrm{GHz}$, respectively.

The path loss exponent $\alpha$ is chosen as 2, which is a generally accepted value. Additionally, the small-scale fading for the channel between the RIS and receiver is modeled by Rice distribution with the shape parameter of $K=10$ in order to allow a few non-line-of-sight (NLOS) paths. Last, the effective noise power for the overall system is chosen as $-100 \mathrm{~dB}$. Table III summarizes the parameters employed in calculating the free-space path loss.

\section{B. Downlink Performance Analysis}

Here we investigate the downlink performance of satellite IoT networks in terms of achievable data rate in three cases: (i) without RIS, (ii) RIS broadcasting, and (iii) RIS beamforming. First, we compare the achievable data rate for conventional satellite systems with RIS-assisted satellites in Fig. 3(a) for Cband. The simulation results show that RIS-assisted satellites provide much higher capacity than conventional satellites, regardless of which scheme they use. In fact, the RIS broadcasting scheme is shown to provide an achievable rate of up to $10^{4}$ times higher than the case without RISs. RIS beamforming is shown to provide a rate of up to $10^{5}$ times higher. In other words, the higher capacity can be achieved with a lower transmit power by using RIS units on satellites. Next, we investigate the impact of the number of RIS elements in Fig. 3(b).

\footnotetext{
${ }^{3}$ Here, the normalized radiation pattern is given only for the transmit antenna. It should be noted that this expression can also be utilized for the receive antenna.
} 


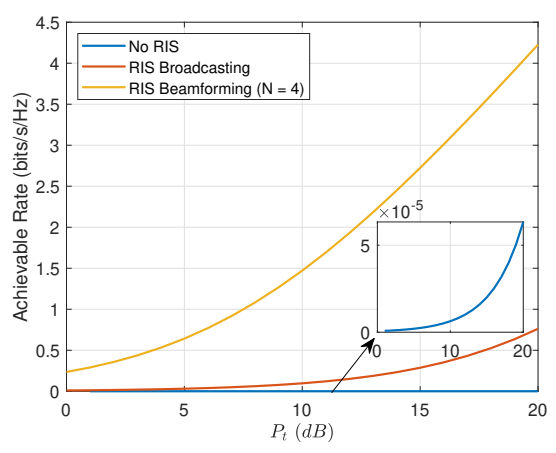

(a)

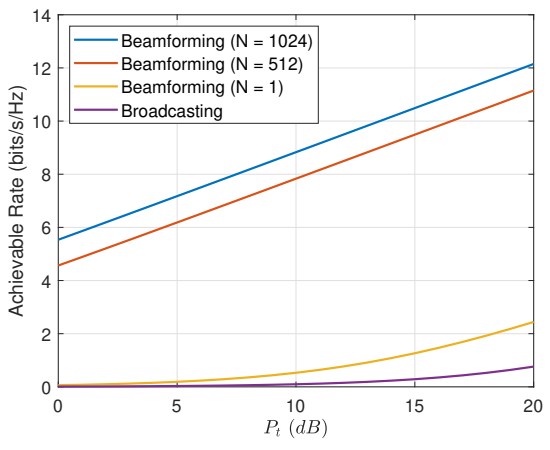

(b)

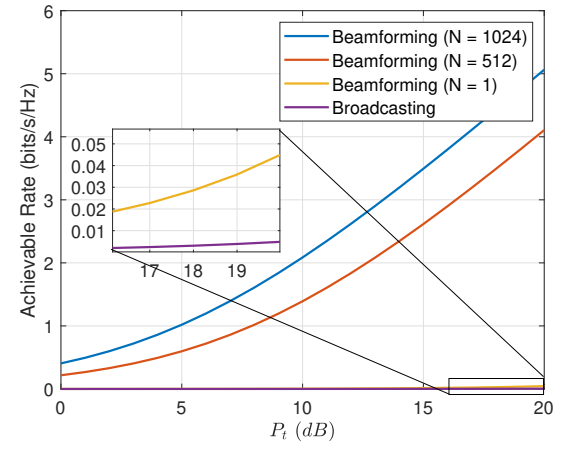

(c)

Fig. 3. Achievable downlink rate relative to transmit power $P_{t}$ for (a) the comparison between the case without RISs and RIS-assisted satellites, (b) the various number of RIS elements in C-band, and (c) the various number of RIS elements in X-band. It should be noted that the elevation angle is $\frac{\pi}{2}$.

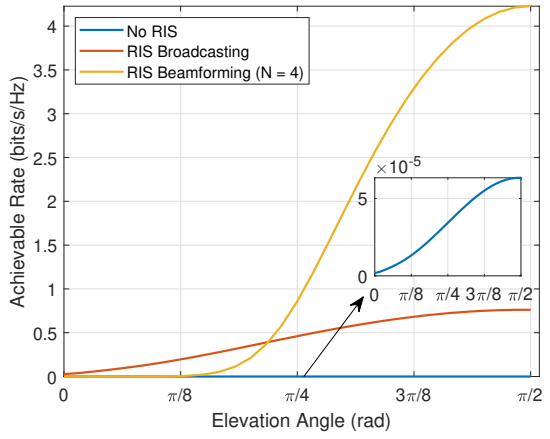

(a)

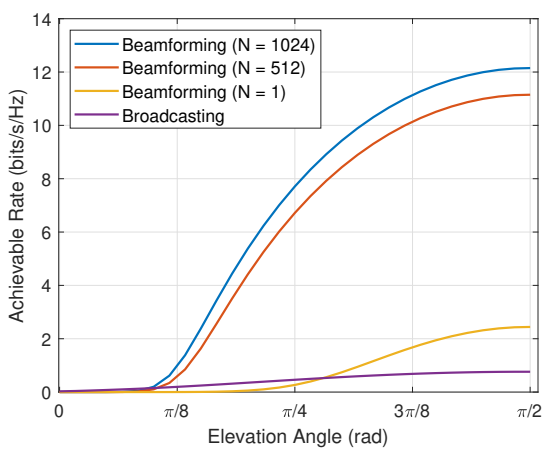

(b)

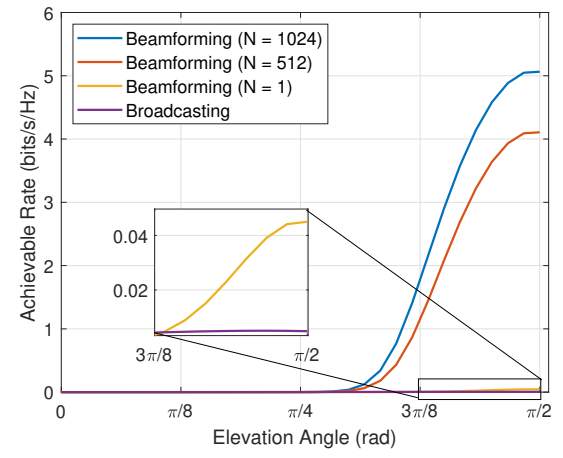

(c)

Fig. 4. Achievable downlink rate relative to elevation angle $\varphi$ for (a) the comparison between the case without RISs and RIS-assisted satellites, (b) the various number of RIS elements in C-band, and (c) the various number of RIS elements in X-band. It should be noted that the transmit power is $100 \mathrm{~W}$.

TABLE III

PARAMETERS FOR FREE-SPACE PATH LOSS CALCULATION FOR RISS AND ANTENNAS OPERATING AT C-BAND AND X-BAND.

\begin{tabular}{lcc}
\hline \hline Parameters & $\mathbf{4 . 2 5} \mathbf{~ G H z}$ & $\mathbf{1 0 . 5} \mathbf{~ G H z}$ \\
\hline$d_{t x}$ & $1 \mathrm{~m}$ & $1 \mathrm{~m}$ \\
$d_{r x}$ & $d$ & $d$ \\
$\alpha$ & 2 & 2 \\
$d_{x}[11]$ & $0.012 \mathrm{~m}$ & $0.01 \mathrm{~m}$ \\
$d_{y}[11]$ & $0.012 \mathrm{~m}$ & $0.01 \mathrm{~m}$ \\
$G_{t}[11]$ & $14.5 \mathrm{~dB}$ & $21 \mathrm{~dB}$ \\
$G_{r}[11]$ & $14.5 \mathrm{~dB}$ & $21 \mathrm{~dB}$ \\
$F^{t x}(\theta, \beta)[11]$ & $\cos ^{13}(\theta)$ & $\cos ^{62}(\theta)$ \\
$F^{r x}(\theta, \beta)[11]$ & $\cos ^{13}(\theta)$ & $\cos ^{62}(\theta)$ \\
$\theta^{t x}$ & 0 & 0 \\
$\theta^{t}$ & 0 & 0 \\
$\theta^{r x}$ & $\frac{\pi}{2}-\varphi$ & $\frac{\pi}{2}-\varphi$ \\
$\theta^{r}$ & $\frac{\pi}{2}-\varphi$ & $\frac{\pi}{2}-\varphi$ \\
$K$ & 10 & 10 \\
$N_{0}$ & $-100 \mathrm{~dB}$ & $-100 \mathrm{~dB}$ \\
\hline \hline
\end{tabular}

As the number of RIS elements increases, the achievable capacity for the RIS beamforming case increases, as expected. However, as given in (4), in the case of RIS broadcasting, the number of elements does not affect the performance. Due to specular reflection, RIS beamforming even with a single element can achieve a higher data rate than the broadcasting case. The main reason for this is the scattering of energy over a wide area. The broadcasting scheme can support more IoT devices than the beamforming scheme because of the larger coverage area provided by the broadcasting. Last, as rain attenuation coefficients and the normalized radiation pattern depend heavily on the operating frequency. The simulation results are shown in Fig. 3(c). For large $N$ values, the achievable rate decreases to less than half, while the ratio of performance loss increases as the number of elements decreases. For example, in the case of a single element, the achievable rate decreases to almost one-fourth of that in the C-band.

As LEO satellites orbit the Earth, the distance between them and the ground stations varies depending on the elevation angle. Distance is one of the major contributors to free-space path loss. Therefore, we have performed the simulations by considering varying elevation angles between zero and $\frac{\pi}{2} \mathrm{rad}$. Fig. 4 shows that the RIS beamforming performs better than broadcasting and the case without RISs. Since $\theta^{r x}$ and $\theta^{r}$ are defined as $\theta^{r x}=\theta^{r}=\frac{\pi}{2}-\varphi$, the elevation angle changes the normalized received radiation pattern. Therefore, with fewer RIS elements at lower elevation angles, the RIS broadcasting scheme provides a slightly better achievable rate than RIS broadcasting. However, increasing the number of RIS elements compensates for the path loss increase owing to the increased distance and decreased received radiation pattern as seen in Fig. 4(b) and Fig. 4(c). 


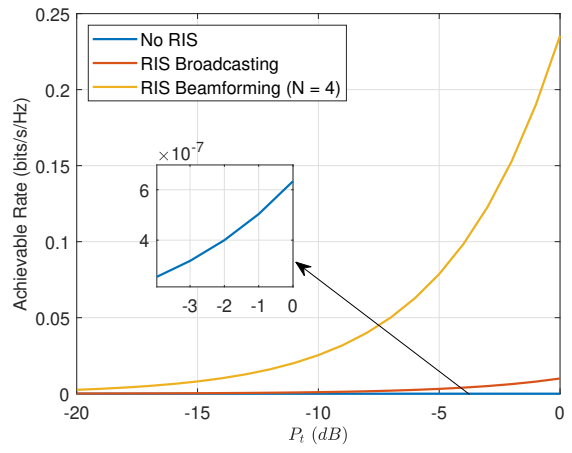

(a)

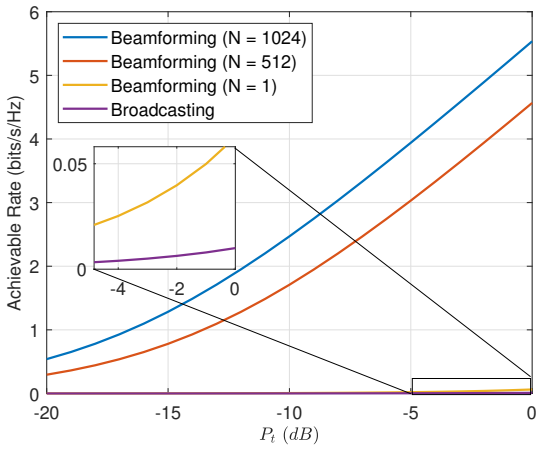

(b)

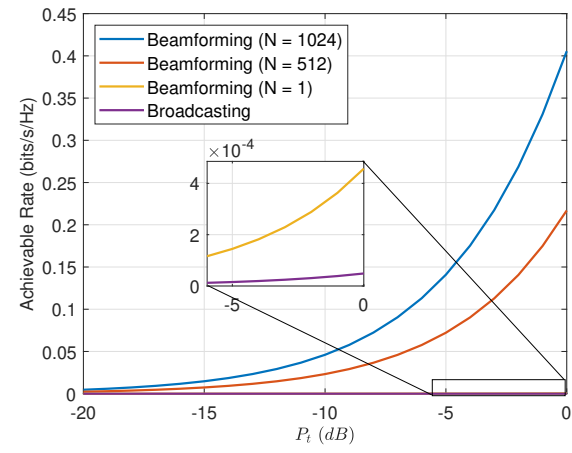

(c)

Fig. 5. Achievable uplink rate relative to transmit power $P_{t}$ for (a) the comparison between the case without RISs and RIS-assisted satellites, (b) the various number of RIS elements in C-band, and (c) the various number of RIS elements in X-band. It should be noted that the elevation angle is $\frac{\pi}{2}$.

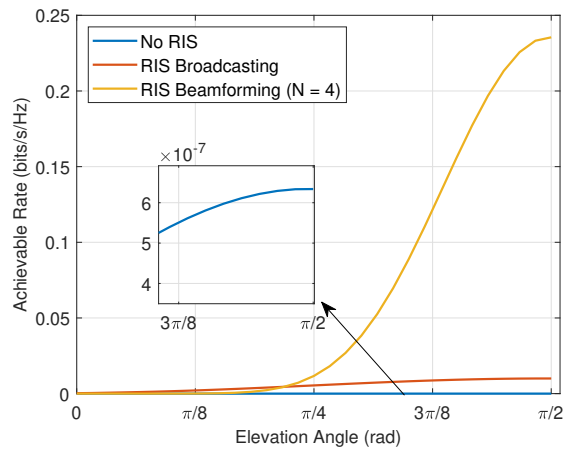

(a)

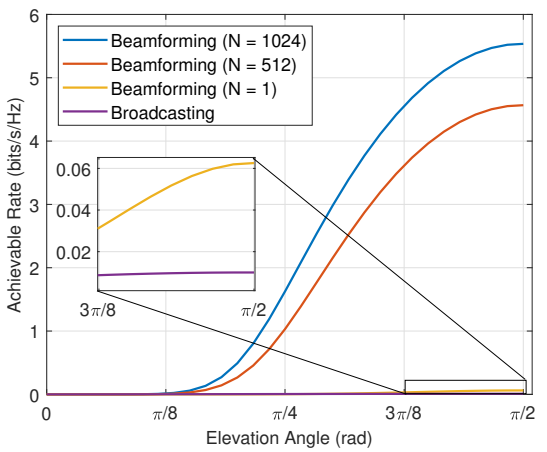

(b)

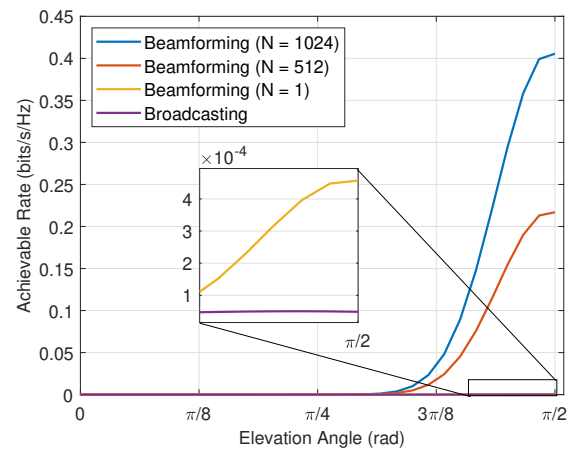

(c)

Fig. 6. Achievable uplink rate relative to elevation angle $\varphi$ for (a) the comparison between the case without RISs and RIS-assisted satellites, (b) the various number of RIS elements in C-band, and (c) the various number of RIS elements in X-band. It should be noted that the transmit power is 1 W.

\section{Uplink Performance Analysis}

In this section, we analyze the uplink capacity of satellite IoT networks in three cases: (i) without RIS, (ii) RIS broadcasting, and (iii) RIS beamforming. As with the analyses of the downlink in the previous section, we first consider the advantage of using RISs in satellites for IoT networks. Fig. 5(a) shows that RIS beamforming can provide an uplink rate that is $10^{5}$ times higher than the case without RIS. This result indicates that the battery and lifetime of battery-limited IoT devices can be significantly increased with RIS-assisted satellites. Moreover, Fig. 5(b) shows that it is possible to obtain $3 \mathrm{~dB}$ more transmit power gain by doubling the number of RIS elements (from 512 to 1024) for the same achievable data rate value. As we can see in Fig. 5(c) that the uplink data rate decreases significantly when the operating frequency increases. As the number of RIS elements increases, the rate of decrease in the achievable rate due to the increase in frequency degrades. Although increasing the operating frequency reduces the achievable data rate performance, the higher frequency can be employed for small IoT devices, which cannot accommodate larger antennas. Fig. 6(a) shows that the RISbeamforming scheme can offer higher performance over a wider range of elevation angles than RIS broadcasting and non-RIS satellites. As we can see in Fig. 6(b), increasing the number of RIS elements marginally improves the operation range in terms of elevation angle. The higher frequency limits the communications in a narrow elevation angle range as shown in Fig. 6(c), which means that the communication duration in the period of a satellite is very short. Hence, using higher frequency may cause many handovers between satellites in a short while.

While the computational capacities and batteries of IoT devices are limited, using RISs can extend battery life since RISs can decrease the computational cost and transmit power for IoT devices. RIS-assisted satellite systems have a twofold advantages for IoT networks. First, RISs can provide higher capacity with lower transmission power. Second, they enable complex operations to be performed in an external environment (i.e. propagation medium) rather than on the devices.

\section{Open IsSUes AND RESEARCh DiRECTIONS}

Although there are many open issues for RIS-assisted wireless communications, we focus here on three of the most crucial issues for RIS-assisted satellites for IoT networks. These are the channel estimation RIS deployment on satellites, and simultaneous wireless information and power transfer (SWIPT), with a focus on the physical layer.

\section{A. Channel Estimation}

Since RISs can change the amplitude and/or phase of the incident electromagnetic wave, they can largely eliminate 
the randomness of the propagation medium. However, RISs must have high-quality channel state knowledge to achieve maximum performance. Channel estimation thus plays a critical role in satellite communications for IoT networks in satisfying the desired QoS. Deep learning may be used for this purpose in order to achieve high efficiency under convincing channel conditions in channel estimation. In a recent work on channel estimation in RIS-assisted backhaul communications, we proposed a channel estimation framework based on graph attention networks (GATs) in [31]. By considering unseen nodes into consideration, the GAT can reduce computational complexity and improve learning performance. In the training process, the obtained signal samples obtained along with known pilot samples were assigned to the nodes and vertices of graphs, respectively. Since IoT devices lack the hardware required for training and using the deep learning model, it is reasonable to deploy the proposed GAT model to LEO satellites rather than IoT devices. By utilizing channel reciprocity, the channel coefficients can be obtained through the uplink pilot signaling. Without the need for downlink pilot signaling, phase adjustment can be performed by the RIS by utilizing the coefficients found for the uplink channel. However, it should be noted that this channel estimation cannot be employed in frequency division duplex communications for uplink and downlink. When using time division duplex, the signal processing can be performed through the propagation environment and RIS rather than IoT devices, thus extending battery life.

\section{B. RIS Fabrication and Deployment}

In this study, we have assumed that RISs are ideal devices that can support lossless reflection and continuous phase shifting. Although this assumption would not entirely hold good in practical application, it is important in terms of showing the upper performance of the system. Our recent study [32] investigated the performance of channel estimation and phase configuration for nonideal RISs in satellite-IoT systems. It was shown that RISs with a sufficient number of discrete phase levels (e.g. 3-bits) can perform almost as well as continuous phase RISs. Also, environmental conditions must be considered in the design of RISs. RISs that can be resilient to temperature variations between day and night should be developed. Although space may indeed be a vacuum, it is also filled with plasma and other particles emitted from the Sun. The electronic components of the communication unit may be affected by charged particles encountered by spacecraft in the Van Allen Belts. As a result, it is crucial to consider radiationresistant RISs and any other innovations that would allow RISs to remain operational under such conditions.

How to implement RISs in LEO satellites is undeniably the first and the most fundamental question. Clearly this is a significant design challenge that needs to be addressed. In particular, depending on variations in the positioning and elevation angle of the RIS units, the device may shadow any or all of the meta-atoms. Moreover, as discussed above, the position of RIS units with respect to the transmit antenna (i.e., $\theta_{n}^{t x}=\theta_{n}^{t}=0$ ) is important to maximizing the performance of the system. Thus, RIS deployment should be carefully considered. Based on the development of their structures, the authors envisage that conformal metasurfaces can be used in the coating of objects with irregular surfaces and arbitrary shapes. It is worth recalling that satellite systems have already utilized reflectarray antennas to reflect the incident beam with a constant phase [33]. These reflectarrays can be replaced by RISs. This would allow satellites to reflect or scatter incident waves with various phase configurations. Interdisciplinary studies can make novel RIS designs possible. For example, it may be possible to place RIS units over a wide area by covering the lower faces of the satellite solar panels. Moreover, the antenna subsystems currently on satellites might be replaced by RISs.

\section{Simultaneous Wireless Information and Power Transfer}

As IoT nodes are power-limited devices, energy harvesting can be considered in a SWIPT framework. RIS-aided SWIPT has already been proposed for achieving significant performance gains in energy harvesting [34]. SWIPT can also be a solution to keeping power-constrained IoT systems running for a long time. But, its applications in satellite-IoT systems are lacking in the literature. Considering the power transfer capacity of space solar satellites with microwave waves [35], the joint utilization of RISs and rectenna arrays can further improve the energy-efficiency of IoT networks. To put it briefly, RIS-assisted satellites for SWIPT in IoT networks deserve more attention in the future.

\section{CONCLUding REMARKS}

Ubiquitous connectivity and user-centric communications are the strict requirements of $6 \mathrm{G}$ networks. As the number of connected devices and IoT networks increases, LEO satellite networks will gain increasing as the backhaul/fronthaul connectivity solutions for densely deployed IoT sensors. However, current systems either suffer from high path loss or require steerable antennas on IoT devices. Given these drawbacks, we introduced an RIS-assisted LEO satellite framework for energy-efficient IoT networks in this study. The motivation behind the RIS communications in satellite-IoT was given using extensive numerical results throughout the study. The potential gain in terms of the transmission powers of lightweight IoT nodes were quantified. Furthermore, open issues regarding the proposed system model were also discussed.

\section{REFERENCES}

[1] Huawei, "Touching an intelligent world," https://bit.ly/38KHhmT, 2019, (Accessed on 01/07/2021).

[2] McKinsey Global Institute, "The Internet of Things: Mapping the value beyond the hype," https://mck.co/3igecTk, June 2015, (Accessed on 01/08/2021).

[3] Deloitte, "The Internet of Things: a technical primer," https://bit.ly/ 35HPPc8, 2018, (Accessed on 01/08/2021).

[4] S. Kota and G. Giambene, "Satellite 5G: IoT use case for rural areas applications," in International Conference on Advances in Satellite and Space Communications, 2019, pp. 24-28.

[5] C. A. Hofmann and A. Knopp, "Direct access to GEO satellites: An Internet of Remote Things technology," in IEEE 5G World Forum, 2019, pp. 578-583.

[6] R. Barbau, V. Deslandes, G. Jakllari, J. Tronc, J.-F. Chouteau, and A.-L. Beylot, "NB-IoT over GEO satellite: performance analysis," in Advanced Satellite Multimedia Systems Conference and the Signal Processing for Space Communications Workshop, 2020, pp. 1-8. 
[7] Z. Qu, G. Zhang, H. Cao, and J. Xie, "LEO satellite constellation for Internet of Things," IEEE Access, vol. 5, pp. 18 391-18 401, 2017.

[8] S. Cluzel, L. Franck, J. Radzik, S. Cazalens, M. Dervin, C. Baudoin, and D. Dragomirescu, "3GPP NB-IoT coverage extension using LEO satellites," in IEEE Vehicular Technology Conference, 2018, pp. 1-5.

[9] E. Basar, M. Di Renzo, J. De Rosny, M. Debbah, M.-S. Alouini, and R. Zhang, "Wireless communications through reconfigurable intelligent surfaces," IEEE Access, vol. 7, pp. 116753-116773, 2019.

[10] L. Dai, B. Wang, M. Wang, X. Yang, J. Tan, S. Bi, S. Xu, F. Yang, Z. Chen, M. Di Renzo et al., "Reconfigurable intelligent surface-based wireless communications: Antenna design, prototyping, and experimental results," IEEE Access, vol. 8, pp. 45913-45 923, 2020.

[11] W. Tang, M. Z. Chen, X. Chen, J. Y. Dai, Y. Han, M. Di Renzo, Y. Zeng, S. Jin, Q. Cheng, and T. J. Cui, "Wireless communications with reconfigurable intelligent surface: Path loss modeling and experimental measurement," arXiv preprint arXiv:1911.05326, 2019.

[12] D. González-Ovejero, O. Yurduseven, G. Chattopadhyay, and N. Chahat, "Metasurface Antennas: Flat Antennas for Small Satellites," CubeSat Antenna Design, pp. 255-313, 2020.

[13] O. Yurduseven, S. Podilchak, and M. Khalily, "Towards Holographic Beam-Forming Metasurface Technology for Next Generation CubeSats," in International Conference on UK-China Emerging Technologies, 2020, pp. $1-4$.

[14] J. Wang, Y. Li, Z. H. Jiang, T. Shi, M.-C. Tang, Z. Zhou, Z. N. Chen, and C.-W. Qiu, "Metantenna: When metasurface meets antenna again," IEEE Trans. Antennas Propag., vol. 68, no. 3, pp. 1332-1347, 2020.

[15] D. Rotshild and A. Abramovich, "Wideband reconfigurable entire $\mathrm{Ku}$ band metasurface beam-steerable reflector for satellite communications," IET Microwaves, Antennas \& Propagation, vol. 13, no. 3, pp. 334-339, 2019.

[16] K. Tekbiyık, G. K. Kurt, A. R. Ekti, and H. Yanikomeroglu, "Reconfigurable intelligent surfaces in action for non-terrestrial networks," arXiv preprint arXiv:2012.00968, 2020.

[17] Q. Wu and R. Zhang, "Intelligent reflecting surface enhanced wireless network via joint active and passive beamforming," IEEE Trans. on Wirel. Commun., vol. 18, no. 11, pp. 5394-5409, 2019.

[18] 3GPP, "Study on Narrow-Band Internet of Things (NBIoT)/enhanced Machine Type Communication (eMTC) support for Non-Terrestrial Networks (NTN)," 3rd Generation Partnership Project (3GPP), Technical Report 36.763, 01 2021, release 17. [Online]. Available: https://portal.3gpp.org/desktopmodules/ Specifications/SpecificationDetails.aspx?specificationId $=3747$

[19] C. Huang, A. Zappone, G. C. Alexandropoulos, M. Debbah, and C. Yuen, "Reconfigurable intelligent surfaces for energy efficiency in wireless communication," IEEE Trans. on Wirel. Commun., vol. 18 no. 8, pp. 4157-4170, 2019.

[20] S. W. Ellingson, "Path loss in reconfigurable intelligent surface-enabled channels," arXiv preprint arXiv:1912.06759, 2019.
[21] C. A. Balanis, Antenna Theory: Analysis and Design. John Wiley \& Sons, 2016.

[22] ITU-R, "Propagation data and prediction methods required for the design of Earth-space telecommunication systems," International Telecommunication Union (ITU), Recommendation P.618-13, 12 2017. [Online]. Available: https://www.itu.int/dms_pubrec/itu-r/rec/p/ R-REC-P.618-13-201712-I!!PDF-E.pdf

[23] —, "Specific attenuation model for rain for use in prediction methods," International Telecommunication Union (ITU), Recommendation P.838-3, 03 2005. [Online]. Available: https://www. itu.int/dms_pubrec/itu-r/rec/p/R-REC-P.838-3-200503-I!!PDF-E.pdf

[24] — , "Characteristics of precipitation for propagation modelling," International Telecommunication Union (ITU), Recommendation P.8377, 06 2017. [Online]. Available: https://www.itu.int/dms_pubrec/itu-r/ rec/p/R-REC-P.837-7-201706-I!!PDF-E.pdf

[25] G. Maral, M. Bousquet, and Z. Sun, Satellite communications systems: systems, techniques and technology. John Wiley \& Sons, 2020.

[26] ITU-R, "Rain height model for prediction methods," International Telecommunication Union (ITU), Recommendation P.839-4, 09 2013. [Online]. Available: https://www.itu.int/dms_pubrec/itu-r/rec/p/ R-REC-P.839-4-201309-I!!PDF-E.pdf

[27] C. Wang, L. Liu, H. Ma, and D. Xia, "A joint optimization scheme for hybrid MAC layer in LEO satellite supported IoT," IEEE Internet Things J., 2021.

[28] J. A. Fraire, S. Céspedes, and N. Accettura, "Direct-To-Satellite IoT-A Survey of the State of the Art and Future Research Perspectives," in International Conference on Ad-Hoc Networks and Wireless. Springer, 2019, pp. 241-258.

[29] N. Letzepis and A. J. Grant, "Capacity of the multiple spot beam satellite channel with Rician fading," IEEE Trans. Inf. Theory, vol. 54, no. 11, pp. 5210-5222, 2008.

[30] L. You, K.-X. Li, J. Wang, X. Gao, X.-G. Xia, and B. Ottersten, "Massive MIMO transmission for LEO satellite communications," IEEE J. Sel. Areas Commun., vol. 38, no. 8, pp. 1851-1865, 2020.

[31] K. Tekbıyık, G. K. Kurt, C. Huang, A. R. Ekti, and H. Yanikomeroglu, "Channel estimation for full-duplex RIS-assisted HAPS backhauling with graph attention networks," arXiv preprint arXiv:2010.12004, 2020, (accepted for the presentation in IEEE ICC2021).

[32] K. Tekbıyık, G. K. Kurt, A. R. Ekti, and H. Yanikomeroglu, "Graph Attention Networks for Channel Estimation in RIS-assisted Satellite IoT Communications," arXiv preprint arXiv:2104.00735, 2021.

[33] J. Huang, "Reflectarray antenna," Encyclopedia of RF and Microwave Engineering, 2005.

[34] Q. Wu and R. Zhang, "Weighted sum power maximization for intelligent reflecting surface aided SWIPT," IEEE Wireless Commun. Lett., vol. 9, no. 5, pp. 586-590, 2019.

[35] C. A. M. Bergsrud, R. Bernaciak, S. Chaieb, and J. Casler, "Rectenna array equipped on satellites," Journal of Spacecraft and Rockets, vol. 53, no. 3, pp. 480-493, 2016. 